\title{
A study of obstetric acute renal injury
}

\section{Aditi Chitale, Kunaal K. Shinde*, Hemant Damle}

Department of Obstetrics and Gynecology, Smt. Kashibai Navale Medical College and General Hospital, Narhe, Pune, Maharashtra, India

Received: 10 June 2018

Accepted: 15 June 2018

\section{* Correspondence:}

Dr. Kunaal K. Shinde,

E-mail: dr.kunaal.shinde@gmail.com

Copyright: (c) the author(s), publisher and licensee Medip Academy. This is an open-access article distributed under the terms of the Creative Commons Attribution Non-Commercial License, which permits unrestricted non-commercial use, distribution, and reproduction in any medium, provided the original work is properly cited.

\begin{abstract}
Background: Acute renal or kidney injury (AKI) is a clinical syndrome characterized by a sudden decline in glomerular filtration rate leading to decreased excretion of nitrogenous waste products. It continues to be a common problem in developing countries. The aim of this study was to understand AKI characteristics in pregnancy and identify the factors related to its unfavorable outcome.

Methods: This prospective study was conducted between January 2014 to December 2017. Out of 1057 patients delivered in our institute during this period, out of which 26 patients with Obstetric AKI were included in this study.

Results: Incidence of obstetric AKI was $2.64 \%$. Their age varied from 19 to 34 years, with an average of 26.2 years. About $21(80.8 \%)$ patients had not received antenatal care. The main causes of AKI were obstetric hemorrhage $(38.46 \%)$ and puerperal sepsis $(15.38 \%)$. The outcome was favorable with complete renal function recovery in $55.76 \%$ patients. Two (7.69 \%) patients became dialysis dependent. Maternal mortality was one $(3.84 \%)$.

Conclusions: Obstetric AKI is a critical situation in developing countries. Lack of antenatal care (80.8\%) is a major contributing factor for obstetric-related complications leading to renal failure. Obstetric hemorrhage $(38.46 \%)$ is the most common cause of obstetric AKI. Late referral in $9(34.61 \%)$, puerperal sepsis in three $(33.33 \%)$, obstetric hemorrhage in three $(33.33 \%)$ and combined sepsis and hemorrhage in two $(27.77 \%)$ are the common contributing factors leading to its unfavorable outcomes as maternal morbidity and mortality. Hence, a multidisciplinary approach is warranted to prevent such an avoidable complication.
\end{abstract}

Keywords: Acute kidney injury, Maternal mortality, Obstetric hemorrhage, Sepsis

\section{INTRODUCTION}

Acute kidney injury is a clinical syndrome characterized by a sudden decline in glomerular filtration rate leading to decreased excretion of nitrogenous waste products like urea, creatinine and other uremic toxins. ${ }^{1}$ Obstetric AKI is usually caused by septic abortions in early pregnancy and by pregnancy toxemia, antepartum hemorrhage, postpartum hemorrhage and HELLP syndrome in late pregnancy. ${ }^{2,3}$ Its incidence has decreased in the developed countries to only $1-2.8 \%$ due to better antenatal care and rare cases of septic abortion in these countries. ${ }^{4,5}$ However, in the developing countries it is still frequent and the incidence is around $4.2-15 \% .^{4}$
High incidence in developing countries is mainly due to limited inaccessibility of antenatal care and emergency obstetric healthcare facilities. ${ }^{6}$ Obstetric AKI may comprise up to $25 \%$ referrals to dialysis centers in these countries and is associated with high maternal and fetal mortality. ${ }^{7}$ Acute tubular necrosis (ATN) is the most common pathology with a good prognosis. ${ }^{7,8}$ There is a high risk of bilateral renal cortical necrosis and consequently of chronic renal failure in these patients. It is an uncommon event and accounts for only $2 \%$ of all cases of AKI with obstetric complications being the most common etiology. ${ }^{3}$ This study was carried out with the objective of finding the factors leading to obstetric AKI and its outcome. 


\section{METHODS}

This prospective study was carried out over a period of 18 months from January 2014 to December 2017. Out of 1057 patients delivered in the institute during this period, out of which 26 patients with Obstetric AKI were included in this study.

\section{Inclusion criteria}

Patients who developed obstetric AKI with oliguria (urine output $400 \mathrm{ml} /$ day) and azotemia (serum creatinine $2 \mathrm{mg} \%)$.

\section{Exclusion criteria}

Patients with pre-existing renal disease or renal insufficiency before pregnancy.

Detailed history of the patients was studied, and their clinical examination and investigations were carried out. Out of the referred cases, the period of referral was considered early if the patient was referred within 5 days of causative event and late if the referral period was more than 5 days. Each patient underwent complete obstetric examination. Specific inquiries were conducted regarding the mode of delivery, need for blood transfusion and surgical intervention. Curettage was performed in patients with retained products of conception as and when required. Renal biopsy was done in patients who required dialysis at the end of 3 weeks. Hemodialysis was performed according to standard indications. Follow-up was done up to 3 months fortnightly after discharge. Outcome was considered favorable as complete recovery if became dialysis independent with good urine output and normal renal function. Patients with improved renal function but not to the normal level and who became dialysis independent at the time of discharge were considered to have partial recovery. Progression to either chronic kidney disease or mortality was considered as an unfavorable outcome. Patients requiring dialysis after 3 months of discharge were considered to have chronic kidney disease.

\section{RESULTS}

Twenty-six patients with obstetric acute kidney injury were observed. This represents obstetric AKI incidence of $2.64 \%$. The mean age of patients was 26 late 2 years. Twelve $(46.15 \%)$ patients were primigravida and $14(53.84 \%)$ were multigravida. About 21 (80.76\%) patients had not received antenatal care. Two $(7.69 \%)$ patients presented in early pregnancy and 24(92.3\%) presented in late pregnancy. Out of 26 patients, $15(57.69 \%)$ had history of preeclampsia. Fourteen $(53.84 \%)$ patients delivered vaginally, 10(38.46\%) underwent cesarean section (CS) and 1(3.84\%) had abortion. One patient underwent obstetrical hysterectomy due to postpartum hemorrhage after vaginal delivery.
Laparotomy was done in $1(3.84 \%)$ patient due to rupture uterus at 30 weeks of gestation. She was primigravida with bicornuate uterus. Mode of delivery in patients with obstetric AKI is shown in Figure 1.

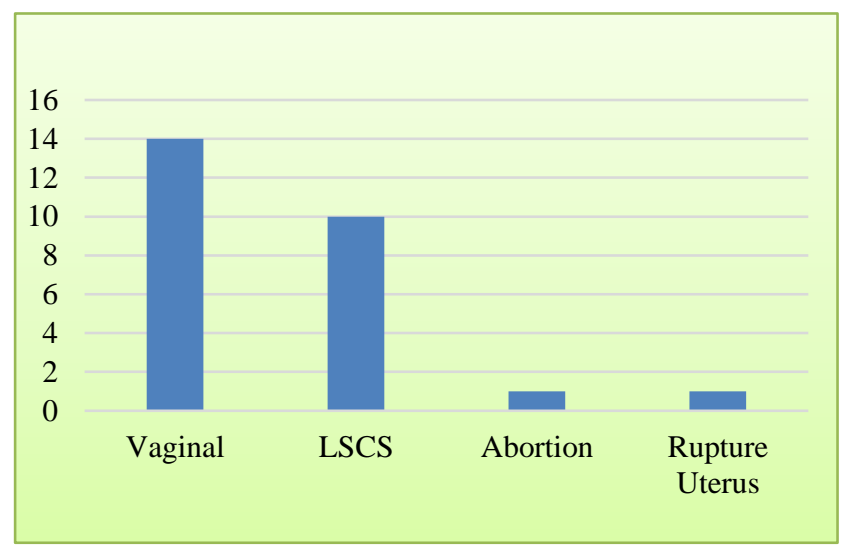

Figure 1: Mode of delivery.

Two $(7.69 \%)$ patients presented with anuria, while 24(92.3\%) presented with oliguria. The mean interval between the causative event and development of AKI was 3.12 days with $\mathrm{SD} \pm 0.963$ and range 3 . The period of referral was less than 5 days in $15(57.69 \%)$ patients, and $11(42.30 \%)$ were referred after 5 days of causative event.

Out of 14 patients referred early, all had complete recovery. Among the patients with late referral, 1(8.3\%) expired, two $(16.66 \%)$ developed chronic kidney disease, one $(8.3 \%)$ had complete recovery and one $(8.3 \%)$ were lost to follow up. Majority $10(83.33 \%)$ of the patients needed dialysis and 4(33.33\%) needed ICU care. Curettage for retained products of conception was required in four $(15.38 \%)$ patients. Fetal mortality was $67.3 \%$. In $10(38.46 \%)$ patients, hemorrhage was the most common etiological factor leading to AKI. APH was the cause in four (15.38\%) and PPH in six (23.07\%) patients.

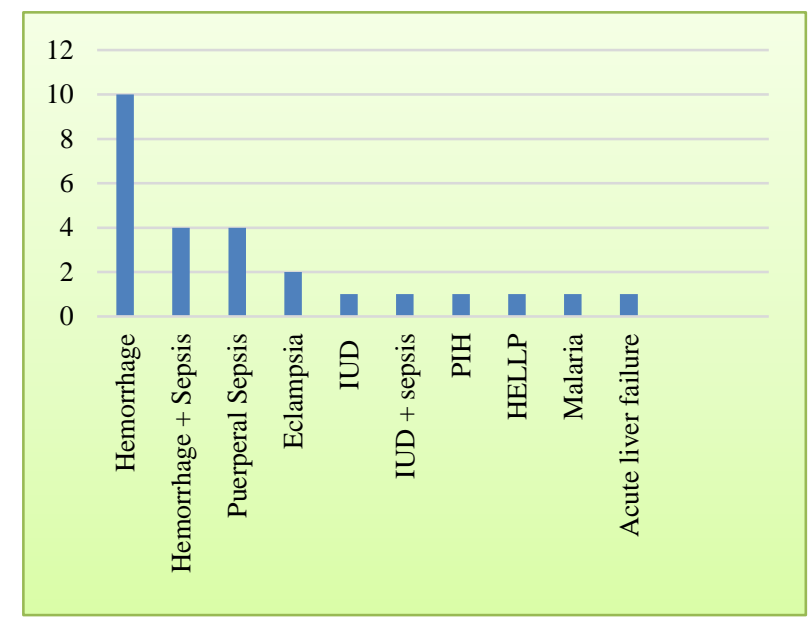

Figure 2: Causes of obstetric AKI. 
Abruptio placentae was the major cause for APH in all these four patients. PPH occurred mainly because of atonic uterus. Puerperal sepsis was diagnosed in four $(15.38 \%)$ patients. Combined hemorrhage and sepsis was found in four $(15.38 \%)$. Two (7.69\%) patients developed AKI due to eclampsia, and intra uterine death (IUD) accounted for the cause of obstetric AKI in $5.77 \%$ (3) patients. Post abortal sepsis as a precipitating event for AKI was present in two $(5.77 \%)$ patients. Other causes like preeclampsia, HELLP syndrome, malaria, IUD with sepsis and acute liver failure (ALF) accounted for $9.61 \%$ of AKI (Figure 2).

Acute fatty liver of pregnancy was the cause leading to ALF. Of the 25(96.1\%) surviving patients who were discharged, $14(56 \%)$ had complete recovery of renal function, $9(36 \%)$ had partial recovery and $2(8 \%)$ required dialysis. On follow-up to 3 months, among the patients with partial recovery, $8(88.88 \%)$ had normal renal functions and Two $(7.69 \%)$ patients who required dialysis at the time of discharge developed chronic kidney disease. Obstetric hemorrhage in two (50\%), sepsis in one $(25 \%)$ and IUD in one $(25 \%)$ were the main causes of chronic kidney disease (Table 1).

Table 1: Number of survival and expired patients at the time of discharge and follow-up.

\begin{tabular}{|ll|}
\hline Status & Patients n=26 $(\%)$ \\
\hline Survived & \\
\hline At discharge & $25(96.1 \%)$ \\
\hline Follow-up & $25(96.1 \%)$ \\
\hline Expired & \\
\hline At discharge & $1(3.84 \%)$ \\
\hline Follow-up & 0 \\
\hline
\end{tabular}

Incidence of maternal mortality was $3.84 \%$. Final outcome of patients with obstetric AKI is shown in Table 2.

Table 2: Final outcome of obstetric AKI patients.

\begin{tabular}{|ll|}
\hline Recovery status & Patients \\
\hline Complete & $20(76.92 \%)$ \\
\hline Chronic kidney disease & $4(15.38 \%)$ \\
\hline Mortality & $1(3.84 \%)$ \\
\hline Lost to follow-up & $1(3.84 \%)$ \\
\hline
\end{tabular}

\section{DISCUSSION}

Incidence of obstetric AKI in the developed countries is $1-2.8 \%$, and in the developing countries, it remains at 9$25 \%$. Present study reported an incidence of $2.64 \%$. Lower incidence in developed countries is due to adequate antenatal care, early diagnosis and timely management of complications. Septic abortion has not been observed any more in these countries. Inadequate antenatal care is a major factor leading to high incidence in developed countries as present study has found that majority $21(80.8 \%)$ patients have not received antenatal care which is comparable to a study done by Khanal et al. where $68 \%$ did not have antenatal checkups. ${ }^{6}$ Obstetric hemorrhage was found to be the major cause of acute kidney injury in this study $(38.46 \%)$, which is similar to studies conducted by Ali et al, Rizwan and Ansari et al. ${ }^{8,10,11}$ Goplani et al found puerperal sepsis as the most common cause for obstetric AKI. ${ }^{4}$ Goplani et al and Ansari et al reported more cases of AKI in late pregnancy than in early pregnancy. ${ }^{4,11}$ Similarly, we found $10 \%$ cases in early pregnancy and $90 \%$ in late pregnancy. This is in contrast to a previous study conducted by Chugh et al in India who reported $59.7 \%$ patients of AKI in early pregnancy. ${ }^{12}$ This major change appears to be due to the legalization of abortion. Present study shows $15.38 \%$ incidence of cortical necrosis with $33.33 \%$ cases in early and $14.89 \%$ in late pregnancy, while it was $23.8 \%$ in another study conducted by Prakash et al with nearly equal incidence in the early (20.5\%) and late (29\%) pregnancies. ${ }^{4}$ This is in contrast to the Western countries where Kleinknecht et al reported $1.5 \%$ incidence of cortical necrosis due to postabortal AKI. ${ }^{13}$ Goplani et al. reported $54.28 \%$ and Rizwan reported 53\% complete recovery rate of obstetric AKI patients. ${ }^{4,10}$ In the present study, authors found similar recovery rate of $55.76 \%$.

Khalil et al reported a maternal mortality rate of $15 \%$ compared to $33.3 \%$ reported by Chaudhri et al ${ }^{14,15}$ Authors found $32.46 \%$ maternal mortality in present study. Recent studies in India have shown a maternal mortality rate around $24 \% .{ }^{16}$ This appears to be the result of aseptic delivery practices and early management of obstetric complications. Present study has shown $7.69 \%$ incidence of chronic kidney disease which is comparable to a study done by Patel et al with $8.4 \%$ incidence of chronic kidney disease. ${ }^{17}$ Short duration of follow-up is the limitation of this study as further long-term follow-up is required to assess the outcome of patients with chronic kidney disease. One important factor which authors have found in this study is that majority $27(51.92 \%)$ patients with complete recovery were referred early while patients with late referral suffered high maternal mortality $4(26.92 \%)$ and morbidity $4(7.69 \%)$. This shows the importance of timely referral of patients with obstetric complications.

\section{CONCLUSION}

Obstetric AKI is a critical situation in developing countries and a rare entity in developed countries. Our study shows that $21(80.8 \%)$ patients had not received antenatal care leading to a major contributing factor for obstetric-related complications leading to renal failure. Obstetric hemorrhage $(38.46 \%)$ is the most common etiology followed by puerperal sepsis (15.38\%). Late referral in $11(34.61 \%)$, puerperal sepsis in six $(33.33 \%)$, obstetric hemorrhage in five $(27.77 \%)$ and combined sepsis and hemorrhage in five $(27.77 \%)$ patients are the common contributing factors leading to its unfavorable outcomes as maternal morbidity and mortality. Though 
maternal mortality has decreased, sepsis still accounts for the majority of deaths.

Funding: No funding sources

Conflict of interest: None declared

Ethical approval: The study was approved by the Institutional Ethics Committee

\section{REFERENCES}

1. Jefferson A, Thurman JM, Schrier RW. Pathophysiology andetiology of acute kidney injury. In: Floege J, Johnson RJ, Feehally J, editors. Comprehensive clinical nephrology. New York:Elsevier Saunders. 2010;806-7.

2. Beaufils MB. Pregnancy. In: Davidson AM, Cameron JS, Grunfeld JP, et al, editors. Clinical nephrology. 3rd ed. New York: Oxford University Press. 2005;1704-28.

3. Prakash J, Tripathi K, Pandey LK, Gadela SR. Renal cortical necrosis in pregnancy-related acute renal failure. J Indian Med Ass. 1996;94(6):227-9.

4. Goplani KR, Shah PR, Gera DN, Gumber M, Dabhi M, Feroz A, et al. Pregnancy-related acute renal failure: A single-center experience. Indian J Nephrol. 2008;18(1): 17.

5. Rani PU, Narayen G, Anuradha G. Changing trends in pregnancy related acute renal failure. J Obstet Gynecol India. 2002;52:36-8.

6. Khanal N, Ahmed E, Akhtar F. Factors predicting the outcome of acute renal failure in pregnancy. $\mathrm{J}$ Coll Physicians Surg Pak. 2010;20(9):599-603.

7. Pertuiset N, Grunfeld JP. Acute renal failure in pregnancy. Baillieres Clin Obstet Gynaecol. 1994;8(2):333-51.

8. Schrier RW. Diseases of kidney and urinary tract. Philadelphia: Lippincott Williams and Wilkins; 2001.
9. Ali A, Zafar S, Mehmood A, Nisar A. Obstetrical acute renal failure from frontier province: a 3 years prospective study. J Postgrad Med Inst (PeshawarPakistan). 2011;18(1)109-16.

10. Rizwan N, Uddin SN. Obstetrical acute renal failure: a challenging medical complication. J Ayub Med Coll Abbottabad. 2011;23(4):66-8.

11. Ansari MR, Laghari MS, Solangi KB. Acute renal failure in pregnancy: one year observational study at Liaquat University Hospital, Hyderabad. J Pak Med Assoc. 2008;58(2):61-4.

12. Chugh KS, Singhal PC, Sharma BK. ARF of obstetric origin. J Obstet Gynecol. 1976;108:253-61.

13. Kleinknecht D, Grünfeld JP, Gomez PC, et al. Diagnostic procedures and long term prognosis in bilateral renal cortical necrosis. Kidney Int. 1973;4:390-400.

14. Khalil MA, Azhar A, Anwar N, Wali R. Aetiology, maternal and foetal outcome in 60 cases of obstetrical acute renal failure. J Ayub Med Coll. 2009;21(4):46-9.

15. Chaudhri N, But GU, Masroor I, Qureshi MA, Shezad MN, Abbasi MSR, et al. Spectrum and short term outcome of pregnancy related acute renal failure among women. Ann Pak Inst Med Sci. 2011;7(2):57-61.

16. Kumar KS, Krishna CR, Siva Kumar V. Pregnancy related acute renal failure. J Obstet Gynecol India. 2006;56(4):308-10.

17. Patel ML, Sachan R, Radheshyam PS. Acute renal failure in pregnancy: tertiary centre experience from north Indian population. Niger Med J. 2013;54(3):191-5.

Cite this article as: Chitale A, Shinde KK, Damle H. A study of obstetric acute renal injury. Int J Reprod Contracept Obstet Gynecol 2018;7:2677-80. 